\title{
X-ray free-electron lasers fire up
}

California's project has the lead as its facility goes live, but Europe aims for its own rapid-fire device.

\section{HAMBURG}

Heinz Graafsma is tired of the "pretty, but useless" images of proteins that regularly adorn the pages of journals such as Nature. "Chemistry depends on changes," says Graafsma, the head of detectors for photon science at DESY, Germany's high-energy physics laboratory in Hamburg. "The static world is boring."

Get ready for the movies. A new generation of light sources - including one just completed in California, one under construction in Japan and one being built outside Graafsma's office - are getting set not only to put atoms and molecules under the spotlight, but also to illuminate their dynamics.

The devices, called X-ray free-electron lasers, produce flashes of X-ray light with angstrom-level wavelengths - small and coherent enough to image individual atoms. The flashes are also more intense than any created before - stuffed with enough photons to create and study extreme states of matter such as plasma.

But perhaps most importantly, the bursts of light are short - just hundreds of femtoseconds long, the time it takes for light to cross a human hair. Pulses as brief as this can record functions, not just forms: the folding of a protein, the action of a catalyst, the splitting of a chemical bond.

"That is the revolutionary thing," says Joachim Stöhr, director of the Linac Coherent Light Source (LCLS) at the SLAC National Accelerator Laboratory in Menlo Park, California. The US\$420-million machine, the first free-electron laser in the world to operate at wavelengths this short, began its first experiments last week.

The new devices will outgun the workhorse of the past half-century: the synchrotron, in which beams of electrons, whipped around in a circle, emit bursts of $\mathrm{X}$-ray radiation. Interest in synchrotrons is still high; the number of users at the four major US synchrotron facilities rose

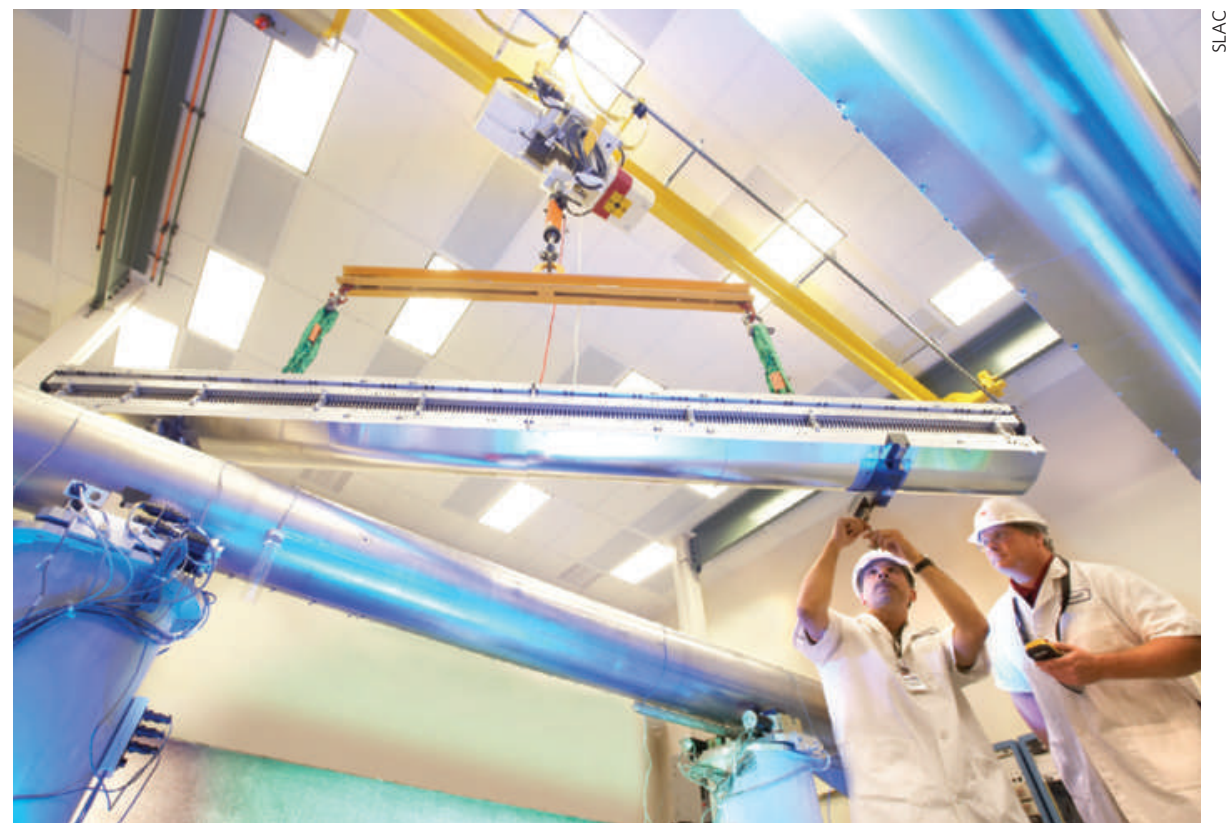

In California, SLAC researchers calibrate the magnets at the Linac Coherent Light Source.

from 6,009 to 8,492 between 2000 and 2008 . But these facilities are starting to reach fundamental limits. Some experiments require many photon 'hits', and these can require weeks, if not months, at even the brightest synchrotrons. In addition, synchrotron pulses are limited to the picosecond regime, a thousand times longer than free-electron laser bursts. Like using a camera with a slow shutter speed, images of always-jittery molecules end up fuzzy.

Just as synchrotron rings were first built for particle smashing, free-electron lasers also depend on a tool borrowed from particle physics: the linear accelerator or 'linac'. The LCLS uses the 43-year-old SLAC linac, in which bunches of electrons are accelerated through a 1-kilometre-long tunnel along a path so tightly focused that Earth's curvature and weak

\section{Science by the femtosecond}

\author{
Atomic physics: exploring \\ how $\mathrm{X}$-rays rip electrons from \\ the inner shells of atoms. \\ Warm dense matter: creating \\ and studying states of matter \\ that lie between solids and \\ plasmas, found in the interiors \\ of planets and cool stars.
}

\section{Single particles and} biomolecules: eliminating the need for crystallography, which is the main bottleneck in describing complex biological structures. Femtochemistry: making movies of chemical bonds being made and broken, of crystals melting and of nanometre-scale droplets nucleating.

Nanometre-scale dynamics in condensed matter: probing how proteins fold and polymers twist. magnetic field have to be taken into account.

The electron bunches then reach a 130-metrelong section of undulators - magnets that 'wiggle' the electrons and coax them into emitting X-rays. The wiggles are tuned to the wavelength of the emitted light, creating a feedback mechanism: electromagnetic fields from the X-rays act on the electrons, concentrating them into small, tight groups that emit amplified light in unison.

On 10 April, LCLS engineers successfully tested this crucial idea, which was first proposed in 1971 (J. M. J. Madey J. Appl. Phys. 42, 1906-1913; 1971). LCLS project director John Galayda remembers seeing a sudden surge in light on that day as the electron beam passed the tenth undulator and the amplification began to occur.

Now it's time to start using the beam. After a summer spent commissioning the first instrument, the first team arrived at 7:30 a.m. on 1 October for five straight days of data collecting. A gas jet shot atoms of neon into the oncoming beam pulses so that the scientists could study what happens when electrons from the atom's innermost shell are stripped away. "It's like peeling an onion from the inside out," says instrument scientist John Bozek.

One of the most anticipated applications for these tiny spotlights (see 'Science by the 
femtosecond') will be imaging single biomolecules. At synchrotrons, proteins have to be crystallized so that the lattice-like structure of many identical proteins clarifies the image made by the relatively incoherent X-rays. But some targets, such as viruses and the proteins found in cell membranes, are notoriously difficult to crystallize.

Expanding the number of described protein structures will be important. But DESY director Helmut Dosch says that more surprises will come from descriptions of how those structures move. Many disorders, such as Alzheimer's disease, arise when there is a problem in the way in which proteins fold. "You have to understand what drives the folding," he says.

There is a drawback to free-electron lasers: there are few chances to work with a piece of the spotlight. At circular synchrotrons, light can be siphoned off to experiment stations at regular intervals - the new PETRA III ring at DESY, for example, has 14 stations that can hold up to 30 instruments. But the straightshot LCLS has just one station holding a single instrument, and work must proceed in series. "The available time is small and the amount of exciting science is large," says Jerome Hastings, head of the LCLS science department. Eventually, SLAC plans to install switching stations, so that light pulses can be diverted to each of six planned experiments.

That's one reason why scientists at DESY think there will still be plenty of work left to do when the European X-Ray Free-Electron Laser (XFEL) is completed in 2014. (Japan also hopes to complete a free-electron laser in 2010, next to its SPring- 8 synchrotron in Harima.) An agreement, expected by the end of this year, will formalize the 13-nation, $€ 1$ 1-1-billion (US $\$ 1.6$ billion) XFEL project.

The XFEL will navigate the terrain beneath urban Hamburg. Germany is footing up to $60 \%$ of the construction costs and is pressing ahead with construction, which began in January.

In August, men in orange coveralls stacked reinforcing bars and electric cabling in a 40-metre-deep chasm, having scooped away hundreds of thousands of cubic metres of dirt and the occasional Second World War mortar shell. From this pit at the edge of DESY, tunnels bearing the linear accelerator will burrow northwest to the town of Schenefeld, 3.4 kilometres away.

The tunnelling is the most expensive component of the XFEL's construction, and much of the 5-year lead that the LCLS has over the XFEL can be attributed to the recycling of SLAC's existing linear accelerator. Galayda says that the Californian project would have cost at least \$300 million more if the team had had to dig a tunnel and build an accelerator from scratch.

But the XFEL has its own trump card: its accelerator will use cryogenically cooled superconducting cavities, allowing the electron bunches to be fired off more quickly. Whereas the LCLS is limited to 120 bursts of light per second, the XFEL will release 30,000.

Massimo Altarelli, XFEL's designate director, says the machine's superior repetition rate will be particularly important in experiments involving dilute targets - a biomolecule floating in a solvent, say - where the chance of registering a photon 'hit' may be slim. But LCLS scientists contend that, most of the time, all of the extra firepower will be wasted: the light will be discarded, absorbed by lead walls.

Stöhr says he is glad that the LCLS is up and running first, but adds that there will be plenty of important science for the XFEL. "There's more than one winner," he says.

Eric Hand

\footnotetext{
In Germany, work is well under way on the European 'XFEL' light source.
}

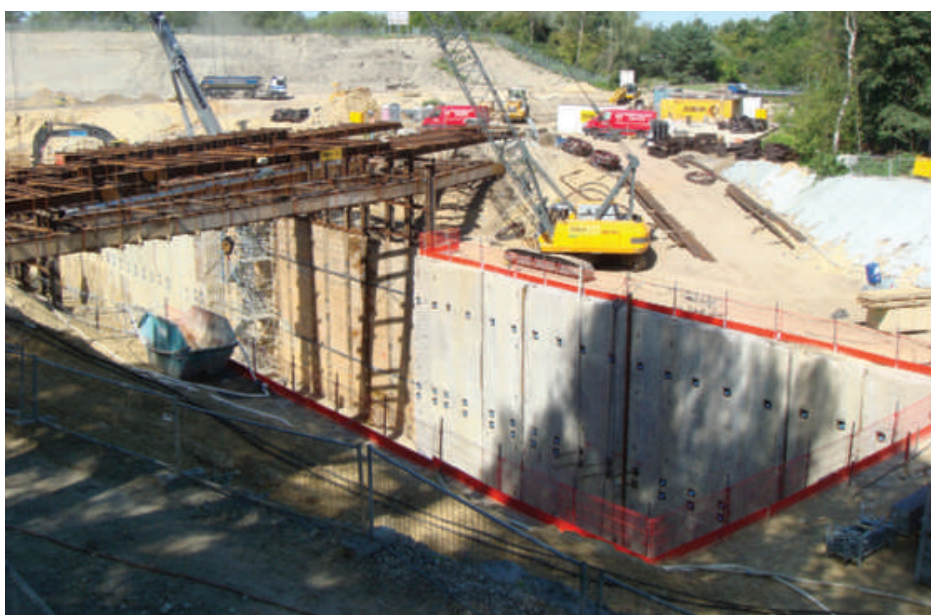

